\title{
A synthesis of recent analyses of human resources for health requirements and labour market dynamics in high-income OECD countries
}

\author{
Gail Tomblin Murphy ${ }^{1}$, Stephen Birch², Adrian MacKenzie ${ }^{1 *}$, Stephanie Bradish ${ }^{1}$ and Annette Elliott Rose ${ }^{1}$
}

\begin{abstract}
Background: Recognition of the importance of effective human resources for health $(\mathrm{HRH})$ planning is evident in efforts by the World Health Organization (WHO) and the Global Health Workforce Alliance (GHWA) to facilitate, with partner organizations, the development of a global HRH strategy for the period 2016-2030. As part of efforts to inform the development of this strategy, the aims of this study, the first of a pair, were (a) to conduct a rapid review of recent analyses of HRH requirements and labour market dynamics in high-income countries who are members of the Organisation for Economic Co-operation and Development (OECD) and (b) to identify a methodology to determine future HRH requirements for these countries.

Methods: A systematic search of peer-reviewed literature, targeted website searches, and multi-stage reference mining were conducted. To supplement these efforts, an international Advisory Group provided additional potentially relevant documents. All documents were assessed against predefined inclusion criteria and reviewed using a standardized data extraction tool.

Results: In total, 224 documents were included in the review. The HRH supply in the included countries is generally expected to grow, but it is not clear whether that growth will be adequate to meet health care system objectives in the future. Several recurring themes regarding factors of importance in HRH planning were evident across the documents reviewed, such as aging populations and health workforces as well as changes in disease patterns, models of care delivery, scopes of practice, and technologies in health care. However, the most common HRH planning approaches found through the review do not account for most of these factors.

Conclusions: The current evidence base on HRH labour markets in high-income OECD countries, although large and growing, does not provide a clear picture of the expected future HRH situation in these countries. Rather than $\mathrm{HRH}$ planning methods and analyses being guided by explicit HRH policy questions, most of the reviewed studies appeared to derive HRH policy questions based on predetermined planning methods. Informed by the findings of this review, a methodology to estimate future HRH requirements for these countries is described.
\end{abstract}

Keywords: HRH planning, Health workforce planning, Health workforce requirements, OECD countries, Highincome countries

\footnotetext{
* Correspondence: adrian.mackenzie@dal.ca

'Dalhousie University, 5869 University Avenue, Halifax, NS B3H 4R2, Canada

Full list of author information is available at the end of the article
} 


\section{Background}

The backbone of any health care system is the human resources who deliver care. Thus, human resources for health $(\mathrm{HRH})$ planning has a direct impact on the functioning of health care systems, which are critical to ensuring a healthy population. According to one of the early seminal texts on the subject, HRH planning is,

the process of estimating the number of persons and the kind of knowledge, skills, and attitudes they need to achieve predetermined health targets and ultimately health status objectives. Such planning also involves specifying who is going to do what, when, where, how, and with what resources for what population groups or individuals so that the knowledge and skills necessary for the adequate performance can be made available according to predetermined policies and time schedules. This planning must be a continuing and not a sporadic process, and it requires continuous monitoring and evaluation. [1].

Put another way, it involves matching the supply of HRH to the requirements for the services they provide [2]. Despite the centrality of $\mathrm{HRH}$ planning to the success of global campaigns such as the Millennium Development Goals [3], it remains a significant challenge. For example, many countries still lack the capacity to maintain accurate counts of their health care providers [4]. Other countries' efforts focus on monitoring HRH supply without considering whether it is adequate to meet HRH requirements [5, 6].

Recognition of the importance of effective HRH planning is evident in efforts by the World Health Organization (WHO) and the Global Health Workforce Alliance (GHWA) to facilitate, with partner organizations, the development of a global HRH strategy for the period 2016-2030 [7]. These efforts build on a number of activities undertaken by the WHO to promote evidence-based HRH planning among member countries. For example, the $\mathrm{HRH}$ Observer series, published by WHO, has included technical issues on tools for modelling $\mathrm{HRH}$ supply and requirements [8], measuring inequalities in the distribution of $\mathrm{HRH}$ [9], and analysing HRH labour markets in developing countries [10], among other topics relevant to $\mathrm{HRH}$ planning.

To inform this process, the HRH unit of the WHO requested that the authors first conduct a rapid review of recent analyses of HRH requirements and labour market dynamics in the member countries of the Organisation for Economic Co-operation and Development (OECD) who are classified as 'high income' by the World Bank. These include Australia, Austria, Belgium, Canada, Chile, the Czech Republic, Denmark, Estonia, Finland, France, Germany, Greece, Hungary, Iceland, Ireland,
Israel, Italy, Japan, Luxembourg, the Netherlands, New Zealand, Norway, Poland, Portugal, Slovakia, Slovenia, Spain, Sweden, Switzerland, the United Kingdom, and the United States (referred to hereafter as the included countries'). Second, it was requested that the authors identify, informed by the findings of the review, a methodology to determine future $\mathrm{HRH}$ requirements for these countries.

The specific objectives of the review were as follows:

(I) To identify all analyses of HRH requirements and health care labour market dynamics for high-income OECD countries published in English within the past 10 years

(II) To categorize the analyses according to the type(s) of models used to estimate requirements, the professions included, the time frames over which they apply, any labour market trends identified, and any assumptions on which they were based

(III) To identify key themes and trends in these analyses emerging over time

(IV) To identify and report gaps in the knowledge base formed by these analyses to inform the development of a global HRH strategy

(V) To identify a methodology to estimate future HRH requirements in OECD countries

\section{Methods}

Multiple steps were taken to obtain all possible relevant analyses of $\mathrm{HRH}$ requirements and labour market dynamics used in the last decade for the included countries. These included a systematic search of published peer-reviewed literature, targeted searches of key $\mathrm{HRH}$ websites, and multi-stage mining of the references of documents obtained through these means. This analysis was undertaken in collaboration with colleagues in the WHO's Health Workforce unit, who contributed to the development of the search parameters, helped identify potentially relevant websites, and invited prospective members to join an international Advisory Group (AG) of $\mathrm{HRH}$ researchers.

The search of the peer-reviewed literature targeted several electronic databases, including PubMed, Informa HealthCare, Web of Science, EconLit, ABI/INFORM, and CINAHL. These were searched for articles whose titles or abstracts contained terms from each of two groups:

1. Any of the following: Health human resources, HHR, human resources for health, HRH, health workforce, health workers, health manpower, doctors, physicians, nurses, midwives, pharmacists, dentists AND

2. Any of the following: planning, forecasting, modeling, requirements, needs, demand, gaps, 
shortage, supply, oversupply, labor market, dynamics, horizon scan, Australia, Austria, Belgium, Canada, Chile, Czech Republic, Denmark, Estonia, Finland, France, Germany, Greece, Hungary, Iceland, Ireland, Israel, Italy, Japan, South Korea, Luxembourg, the Netherlands, New Zealand, Norway, Poland, Portugal, Slovakia, Slovenia, Spain, Sweden, Switzerland, UK, USA, OECD, high-income

Websites of multiple national organizations such as departments of health, bureaus of statistics, and HRHspecific planning bodies were searched, as well as those of international organizations such as the WHO, the GHWA, the OECD, and the World Bank. When search functions were available for website content, the relevant search terms presented above were used. If search functions were not available, sections of these websites titled 'Publications,' 'Reports', or similar were searched for documents pertaining to HRH analyses. A full list of the websites searched is included as Additional file 1 .

The database and website searches yielded over 1000 documents deemed to be potentially relevant. The titles, abstracts, and/or executive summaries of these documents were then reviewed to ensure that they were published in English between March 2005 and March 2015 and included a primary analysis of HRH requirements or labour market dynamics in one or more included countries.

Based on these criteria, 181 documents were selected for full-text review. Although it was not possible within the allotted time to mine the reference lists of all 181 documents, a selection of the 30 documents that cited the most potentially relevant works were mined for additional documents. The initial list of documents resulting from these activities was then circulated to the AG with the request that they identify any relevant works they felt had not yet been included. The search process is illustrated in Fig. 1.

In total, 224 documents were included in the review. A data extraction tool was created to facilitate summarization of key features of each of these documents in terms of their scopes, methods, conclusions, and any major issues or themes identified. A copy of this tool is available as Additional file 2 .

\section{Results}

\section{Jurisdictional focus}

The documents reviewed covered the 32 included countries specifically, with others looking at groups of countries (such as the European Union or WHO regions) or globally (Fig. 2). The United States was the jurisdiction covered by the most documents, with 53 (24\% of all documents) specific to that country, followed by Canada with 27 (12\%). Thirty-two documents (14 \%) focused on multiple

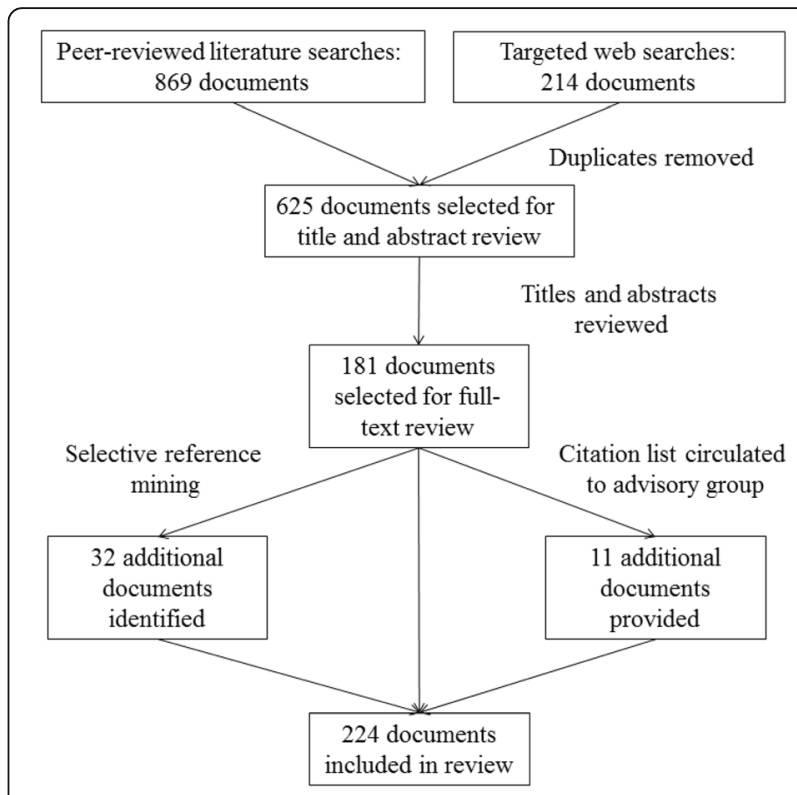

Fig. 1 Search strategy

countries, most commonly the European Union and the OECD with seven documents (3\%) each. Most of the documents focusing on individual countries were those where English is an official language-not surprising given the search parameters-although it is noteworthy that Belgium, Japan, and the Netherlands were each the focus of at least five documents. Other countries for which more than one document was found included Finland (seven), Germany (three), Iceland (two), Israel (two), Italy (two), Norway (three), Portugal (two), Scotland (four), and Wales (two).

\section{Professions included}

A diverse range of health professions were covered in the documents (Fig. 3). Seventy-four documents were not specific to any particular health profession, instead analysing health care system and labour market issues that affect all health professions to varying degrees.

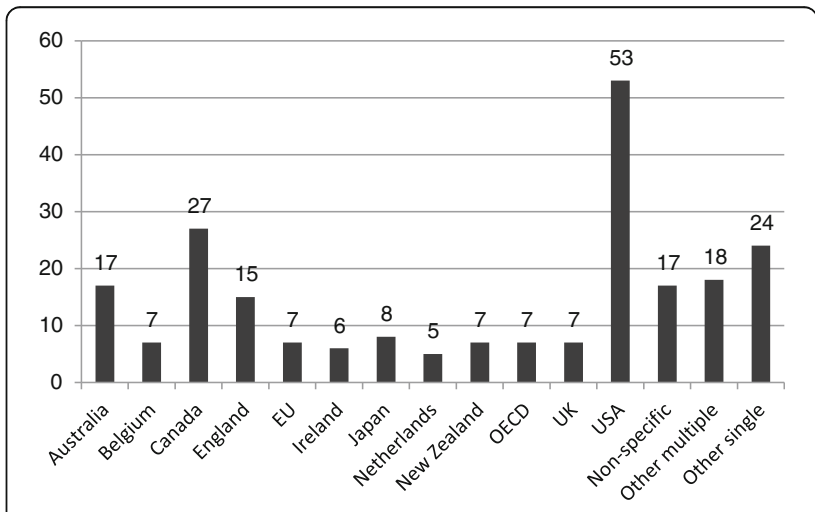

Fig. 2 Number of documents by jurisdiction of focus 


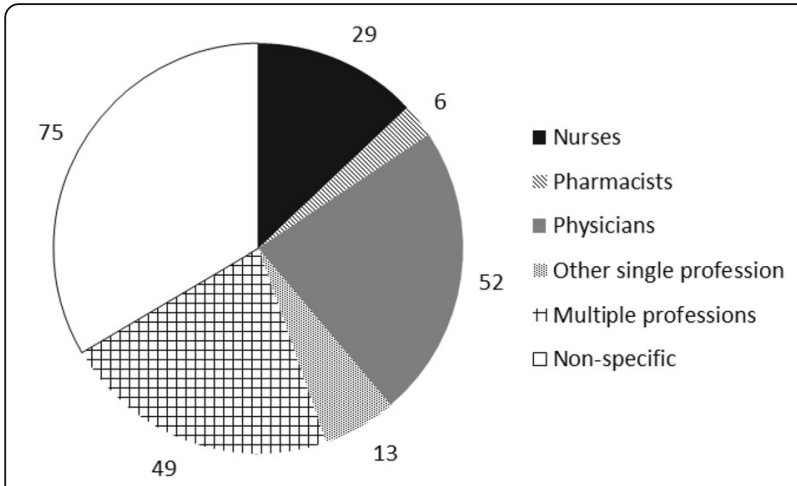

Fig. 3 Number of documents by profession of focus

Among documents focusing on a single profession, 52 (23\%) documents covered physicians, 29 (13\%) covered nurses, and pharmacists were covered in 6 (3\%) documents. The only other professions to be the sole focus of more than one document were dentists (four), midwives (two), and physiotherapists (two).

Although 49 documents included analyses for more than one profession, the majority of these (38) considered those professions in isolation of each other. Only 11 of these included analyses that considered the interdependence between different professions.

\section{Date of publication}

Although the review spanned the past decade, 144 documents (64\% of those included) were published in 2010 or later (Fig. 4). The number of documents published increased annually from 2005 through 2009, with the maximum of 32 published in 2012.

\section{Analytical time frames}

The documents that provided quantitative analyses of HRH supplies or requirements did so over varying time periods (Fig. 5). Sixteen analyses were cross-sectional, providing $\mathrm{HRH}$ analyses for one particular point in time. The others varied widely in the length of time they covered, with 27 documents each including projections 1 to

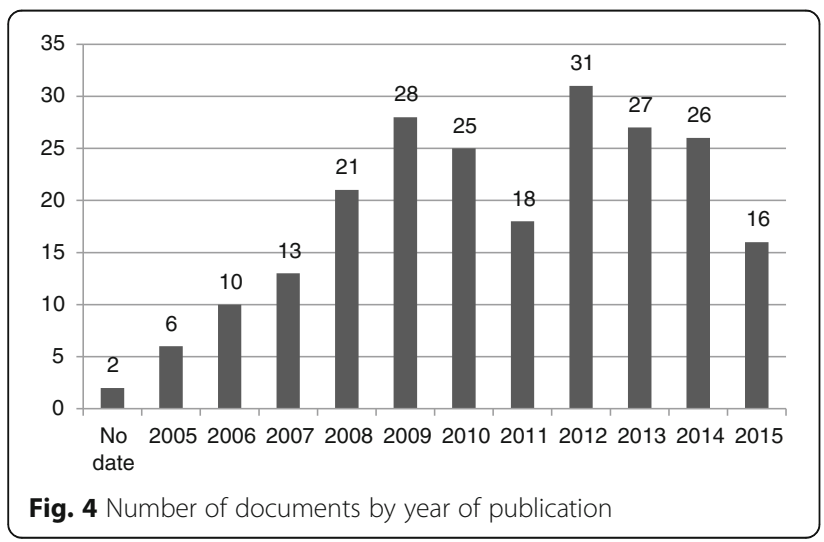

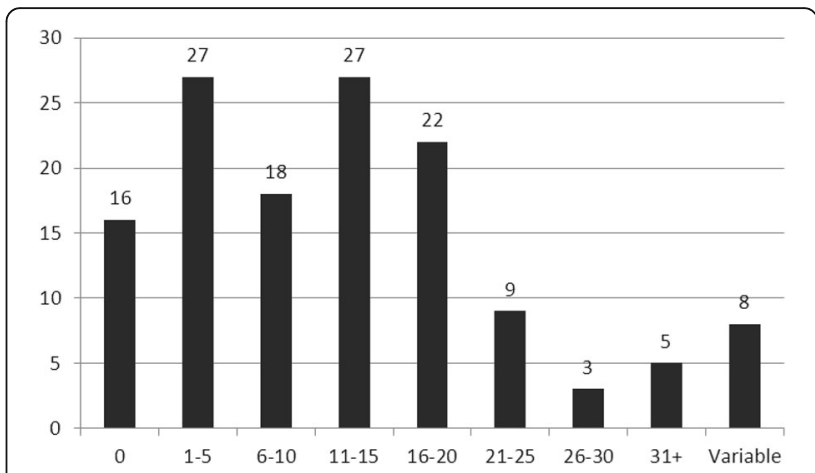

Fig. 5 Number of documents by analytical time frame

5 years ahead and 11 to 15 years ahead. Five documents included projections more than 30 years into the future, while eight used multiple projection periods in their analyses.

\section{Types of models used}

A number of different types of models were used to perform the $\mathrm{HRH}$ analyses in the included documents, which we have grouped into three categories (Fig. 6). These include provider-based (sometimes referred to as supply-based), utilization-based (sometimes referred to as demand-based), and needs-based approaches. Utilization-based approaches are those in which current or target rates of health service utilization are multiplied by estimates of future population size, which are then converted to HRH requirements using productivity estimates. Provider-based approaches are those in which $\mathrm{HRH}$ requirements are estimated primarily by multiplying current or target provider-population ratios to the estimated size of the future population, sometimes adjusting for basic demographic factors like age and sex. Needs-based approaches are those that determine HRH requirements by applying estimated future levels of health in the population to best practices (or current policy) for service provision in response to different

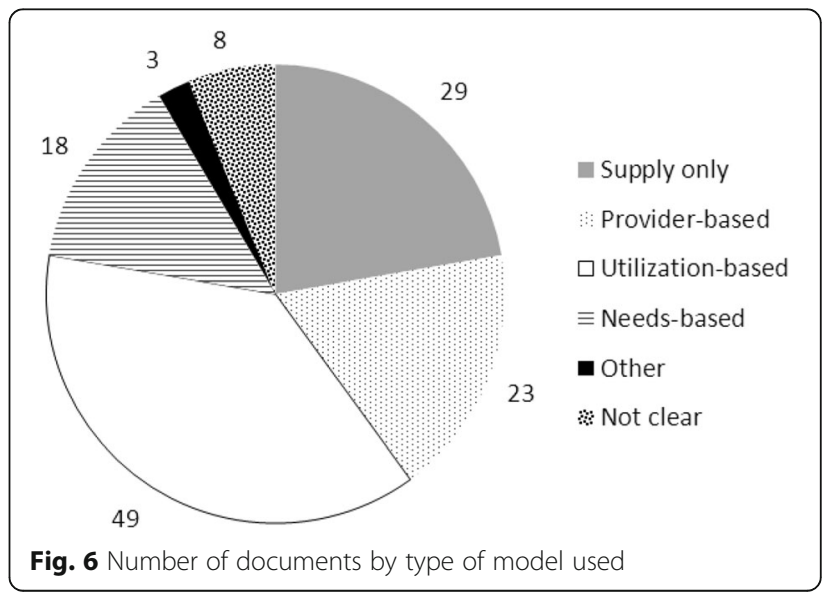


levels of health. Provider requirements are then estimated from the best practice (or current productivity norms) of delivering care to meet those health care needs. It should be noted that the categorization of models in Fig. 6 is based on their characteristics and this typology as opposed to how authors' document may have characterized them (see the 'Results' section for more detail on the different use of terms such as 'need' and 'demand' across documents).

Of the 224 documents reviewed, 130 described quantitative analytical methods and/or analyses of HRH supply or requirements (as opposed to, for example, focusing on a qualitative discussion of HRH labour market or policy issues). Of these, 29 (22 \%) analysed HRH supply only-i.e. without contrasting it with HRH requirements. Among those documents analysing requirements, 49 (38 \%) used utilization-based approaches. Twenty-three (18\%) used provider-based approaches while 18 (14\%) used needs-based approaches. We were not able to determine which type of approach was used in eight documents (6\%).

The approaches described in three documents (2\%) did not fit any of these categories. The primary determinants of HRH requirements described in these documents included the following:

- 'Local health board plans' [11]

- Health needs, willingness/ability to pay, regulatory structures [12]

- Demography, health needs, service levels, productivity, 'clinical care microsystems', 'policy/ governance/power, 'funds and support,' 'individual response:behaviour biology'-several of these are identified as influencing both HRH supply and requirements [13]

\section{Assumptions described}

A detailed analysis of the various assumptions used in each of these planning models is difficult because such assumptions are often implicit. However, the assumption most commonly articulated in these documents was that 'the status quo will continue'-generally that the only model parameters whose value will change over time are those pertaining to population demographics, while other parameters such as service delivery models, practice patterns, productivity, and prevalence rates of various health conditions are assumed to remain constant. Three other explicit assumptions noted in multiple documents were as follows:

- There are no unmet HRH requirements in the base (or first) year of the analysis (see, for example, [14]).

- Utilization of services is a proxy for demand (see, for example, [15]).
- The current ratio of HRH to population is adequate (see, for example, [16]).

Although the assumptions used in these analyses were often not stated explicitly, 13 documents (6\%) included multiple scenarios to demonstrate the sensitivity of their results to different parameter values such as HRH retirements, population growth, or productivity. Each of these demonstrated that projections of future HRH supply and requirements are highly sensitive to different assumptions about how various determinants would change over time.

\section{Trends and issues identified}

A number of trends and issues were raised repeatedly in these documents, many of which have been identified by other recent reviews of HRH studies (e.g. $[5,6,17,18]$ ). An exhaustive list of citations for documents in which each issue is raised would be prohibitively long, but for each of these recurring topics, a few relevant documents are cited as examples. These recurring topics are listed below under two additional subheadings: 'Inputs into $\mathrm{HRH}$ planning' and 'HRH planning methods'.

\section{Inputs into HRH planning}

Aging populations The most frequently raised issue across these documents was the aging of populations. For example, the population aged 65 and older across all OECD countries is expected to increase by over $70 \%$ from 208 million in 2015 to 355 million in 2050, while the number of those aged 80 and over is expected to increase from 56 million to 133 million over the same period [19]. Most of the reviewed documents indicate that this growth in older populations is expected to significantly increase requirements for health care in these countries, because older people tend to use more health care. Other reviewed documents, however, note that this view conflates the relationships between aging, population health, and service requirements They explained that, while the probability of sickness increases with age, population aging is partially reflective of improvements of the average level of health within each age group, and as such, the failure to account for this distinction between demographic and epidemiological shifts results in overestimates of HRH requirements [20-24].

Chronic disease and comorbidities Multiple documents identified a trend of increasing prevalence of multiple chronic conditions as likely to contribute to increased future requirements for HRH (e.g. $[25,26])$. Only a small minority of these, however, included empirical analyses of such trends and their potential impacts on HRH requirements (e.g. [27]). Others emphasize that this change in the complexity of health care needs will require changes in the skill mix of available HRH (e.g. [28]) and/ 
or the models of care delivery they use (e.g. $[5,6,29])$; however, we found no documents that modelled the impacts of changes in needs and potential associated changes in skill mix or care models in terms of their impacts on HRH requirements.

Aging workforces Many of the included documents reported that the stock of available HRH in the jurisdictions to which they pertained is aging, for example, an average of one in three physicians in OECD countries is aged 55 or older [28]. This has prompted concerns about large future HRH shortages, and a number of documents included specific analyses of the potential impacts of different retirement scenarios on HRH supply in their respective jurisdictions (e.g. [29]). In some documents, it is suggested that strategies to encourage HRH to delay retirement be considered as a means of addressing $\mathrm{HRH}$ shortages (e.g. [30]).

HRH migration HRH migration across countries is frequently raised as an issue in these documents and in several cases is the primary focus of the analysis (e.g. $[31,32])$. Perspectives on migration differ across documents, for example, migration is variously identified as a leading cause of (e.g. [33-35, 36]) or important solution to (e.g. [37, 38]) existing and/or projected future imbalances. Others, in contrast, examine the potential impacts of reducing in-migration from other countries, for example, due to concerns about self-sufficiency and the ethics of international recruitment of HRH (e.g. [27, 39]) or in response to perceived HRH surpluses (e.g. [40]).

Distribution of resources The planning models included in the review focus on optimizing the number of $\mathrm{HRH}$ at a particular jurisdictional level; ensuring those resources are appropriately distributed within a given jurisdiction is an important but separate issue. Regardless of the methods used to analyse HRH requirements, many documents noted that estimated surpluses or shortages at one jurisdictional level (e.g. national) may mask shortages or surpluses at other (e.g. state or municipal) levels (e.g. [25, 41]). Several types of $\mathrm{HRH}$ imbalances are discussed. Most often, the imbalance noted is between urban and rural areas (e.g. [34, 42-44]). Other types of imbalances described are those that occur across professions, sectors, and/or specialties, such as between the acute and primary care sectors (e.g. [16, 45-48]).

Interprofessional education and practice A number of documents included in this review have identified that modern health care increasingly requires the competencies of more than one profession so that systems must begin to rely increasingly on multi-professional teams of health care providers $[5,49-51]$. As such, they note the increasing importance of HRH planning models with the capacity for team-based planning as opposed to single professions in isolation (e.g. [5, 6, 16, 47, 52]). However, only a few such models-from Australia [53-56], Canada [57, 58], and New Zealand [29, 59-61] - were found through this review.

Changing care delivery models Although most of the approaches reviewed assume that current care delivery models will continue in the future, a number of documents (e.g. [28, 62-64]) identify a need for HRH planning models that can incorporate or accommodate changes in care delivery models which may be required to address changes in health care needs and/or address those needs in more effective or efficient ways. Comparatively few of the approaches reviewed [29, 53-61] appear to have the capacity to account for such changes.

Changing practice patterns Changing practice patterns or other provider behaviours are identified in some documents as determinants of $\mathrm{HRH}$ requirements. A frequently cited example of such a change is a reduction in the working hours of physicians; other things equal, such reductions reduce the effective supply of physicians and, by extension, the supply of the services they provide. Although decreases in physician working hours are frequently attributed to the growing proportion of female physicians (e.g. [25, 30, 43, 65-67]), other evidence indicates that this reduction in activity is occurring among all physicians, across gender and age groups $[68,69]$.

Evolving scopes of practice and regulatory structures The issue of scopes of practice is addressed in several documents, specifically in terms of expanding scopes of different health professions over time. A specific example involves task-shifting, whereby tasks traditionally performed by one type of HRH (usually relatively expensive and/or scarce, e.g. physicians) are instead delegated to another type of $\mathrm{HRH}$ (usually less expensive and/or scarce, e.g. nurses) [5]. Other things equal, task-shifting reduces requirements for the former profession(s) while increasing them for the latter-such as the expansion of Nurse Practitioner scopes of practice and the potential reduction in demand for physicians [70]. More broadly, it was noted that the evolving scopes of practice for all types require increasing familiarity with a growing range of technologies [47]. Although some approaches include the capacity to adjust for changes in scopes of practice (e.g. $[29,55])$, similar to another recent review of HRH planning approaches [6], we found no examples of such changes actually being accounted for in modelling exercises. In none of the included documents were potentially contracting scopes of practice discussed.

Changes to legislation and the other structures that govern the regulation, management, and delivery of health care are identified as an issue in several documents. An example 
is the Affordable Care Act (ACA) in the United States; one estimate suggests that the ACA will substantially increase demand for nurses [71]. Other examples of regulatory developments likely to impact $\mathrm{HRH}$ labour markets are changes to retirement age in countries such as France [6] and policies aimed at regulating dual practice by physicians [72]. More broadly, it is becoming increasingly recognized that existing national regulatory structures require strengthening to ensure the adoption and implementation of effective $\mathrm{HRH}$ policies (e.g. [12, 73, 74]).

Incentives Several documents (e.g. [72, 75-77]) discuss the importance of provider financial and non-financial incentives as determinants of provider behaviour. Some of these emphasize the importance of considering these issues within the broader economic context in which HRH live and work (e.g. [40, 78-80]). However, relatively few documents (e.g. [49, 71, 76, 81-83]) include quantitative analyses of the relationships between these incentives and aspects of HRH supply (including levels of participation and activity) or requirements (e.g. as influenced by productivity rates). Some documents factor provider rates of pay into future $\mathrm{HRH}$ supply and requirement estimates (e.g. [84-87]); others explicitly investigate the relationship between different incentives and HRH outcomes such as labour force participation and activity (e.g. [81]). It was also emphasized that the effectiveness of various incentives will vary according to different economic contexts [80].

Technological changes Many of the reviewed documents identified technological change as a factor likely to have a significant impact on HRH balance in their respective jurisdictions (e.g. [5, 64, 88]). These include changes in the technology used in health care-such as those allowing for less invasive surgeries [4] —as well as those used by health care consumers, such as mobile phone apps for the management of chronic conditions (e.g. [89]). The impacts of such changes in terms of $\mathrm{HRH}$, however, are not clear from the analyses included in this review. Comparatively few documents directly incorporate consideration of technology changes into quantitative analyses. Among those that do (e.g. $[63,90])$, the exact nature of the changes in question is not made explicit.

Balancing the private and public sectors The health care systems in the included countries incorporate a mix of public and private service providers, and the relative size of these sectors varies considerably across and within countries. The public sector is the focus of the vast majority of the included analyses, although several documents emphasize the need for better data on, and coordination by national planners with, the private health care sectors (e.g. $[6,91,92])$. An example of the challenges in effectively managing both the public and private health care systems was raised in multiple documents (e.g. [72, 82]) discussing the issue of dual-practising clinicians-i.e. those who provide services in both the public and private sectors-noting that policies intended to dissuade them from devoting time to private practice may instead drive them to leave the public sector entirely.

\section{HRH planning methods}

Inconsistent use of terms Terminology differs across documents, with terms such as 'need', 'demand', and 'utilization' used almost interchangeably by some authors. Crettenden and colleagues [93], for example, use utilization as a measure of demand, suggesting that the authors consider these to be equivalent. Other documents note that utilization represents the intersection of supply and demand and, unlike in most markets, demand for health care is not independent of supply, which can result in market failure [2, 72, 78]. Similarly, the Centre for Workforce Intelligence [15] describes its model as estimating demand but uses utilization to measure demand and identifies a needs-based approach [2] as the basis for the model. Other documents, in contrast, emphasize that these are three distinct constructs, none of which can be considered to be a measure of the other (e.g. [2, 72, 94]).

In addition, the term 'shortage' is used to describe different HRH situations in different documents. For example, $\mathrm{HRH}$ shortages are defined in terms of vacant HRH positions (e.g. $[95,96])$ or measured as the degree to which jurisdictional HRH requirements exceed supply (e.g. $[15,65,91])$. Similarly, the presence of unemployment among HRH is deemed by some to reflect a surplus (e.g. [97]) whereas in others a surplus is considered to mean that the jurisdictional supply is greater than requirements (e.g. [86, 98, 99]). Others depict surpluses or shortages as determined at least in part by opinion (e.g. [96, 100, 101]).

Stakeholder engagement The planning approaches identified through this review include a mix of theoretical and applied methods. Among the latter, the importance of stakeholder engagement is acknowledged to varying degrees. Perhaps the most thoroughly documented examples of stakeholder engagement in $\mathrm{HRH}$ planning methods are the elicitation and horizon scanning efforts described by the United Kingdom's Centre for Workforce Intelligence [102, 103]. As part of this approach, multiple groups of stakeholders are engaged in a variety of activities at different stages of the analytical process to help planners identify current and potential future HRH planning issues, articulate potential policy scenarios, supplement administrative data with professional opinions, and interpret and validate results based on their respective experiences. 
Data quality The validity of any HRH planning exercise is contingent upon the availability, relevance, and the accuracy of the data to which planners have access. Needsbased approaches in particular require comprehensive data on not only the supply of HRH but also on the health of the population, planned levels of service provision for different health problems, and HRH productivity, which may not always be available. Despite the online availability of important $\mathrm{HRH}$-related data from organizations such as the WHO and the OECD, almost every document included in this review articulated concerns about inadequacies in the data available to inform HRH planning (e.g. $[3,4,75,86])$.

Iterative planning Many documents (e.g. [2, 5, 6, 64]) emphasized the importance of HRH planning being conducted on an iterative, ongoing basis. This is not only so that planning models can be updated with the most current available data but also so that the relevant stakeholders can be engaged, the validity of any necessary assumptions can be tested, and the effectiveness of implemented strategies can be regularly assessed.

\section{Discussion}

\section{Limitations of this review}

Despite our efforts to assemble a complete set of $\mathrm{HRH}$ analyses pertaining to the included countries, some relevant documents may have been missed for any of the following reasons:

- It was not possible to include documents written in languages other than English. This is a particularly important limitation given that English is not an official language for some countries included in the review.

- Not all potentially relevant websites could be included in the review, and those that were included could not be searched exhaustively.

- It was not possible to mine the references of every relevant document.

- Some relevant peer-reviewed publications may not have been part of the databases searched and/or may not have included any of the search terms used in their titles or abstracts. This may be due to variations in the use of headings and sub-categories in the various databases or in the language used to describe HRH research and planning (see the 'Results' section for examples).

\section{Limitations of the available evidence}

Although many documents have been published describing various aspects of $\mathrm{HRH}$ markets in included countries, they do not collectively present a clear or consistent picture of what the HRH situation is expected to be across-or even within-these jurisdictions in the future. There are several reasons for this:
- The HRH research and/or policy question(s) to be answered by the various analyses are often not clear; different approaches may be required to answer different questions.

- As shown in Fig. 3, the majority of the available studies focus on physicians and nurses; there is little evidence on the supply of-let alone the requirements for-the other $\mathrm{HRH}$ that make up each country's workforce.

- Many of the analyses identified through this review were specific to sub-jurisdictions of the included countries, for example, to one Canadian province [104] or to England [86] as opposed to the entire United Kingdom. Such analyses cannot provide a complete picture of the overall HRH situations in the countries. For most of the jurisdictions included in this review, no quantitative analyses of nationallevel HRH gaps-i.e. the difference between $\mathrm{HRH}$ supply and HRH requirements-were found. For others, only analyses of HRH supply (not requirements) were found, so it is not possible to present a picture of the $\mathrm{HRH}$ situation across the included countries here.

- Even among those analyses that project HRH shortages or surpluses, these are measured in different units-for example, head counts as opposed to full-time equivalents (FTEs) in different studies-they span differing time periods, and are specific to a variety of different sectors (e.g. primary care, mental health services, and long-term care).

- As outlined above, there are important differences in the various approaches used to conceptualize and measure HRH supply, requirements, shortages, and surpluses across these countries. Some of these approaches, for example, describe their approach as measuring $\mathrm{HRH}$ requirements in terms of demand, even though demand in the market for health care is not independent of supply [2,72,94], while others do so in terms of need, which is independent of supply. Given these two fundamentally different concepts, estimates of HRH 'demand' cannot be meaningfully combined with estimates of $\mathrm{HRH}$ needs, since they are likely to address significantly different policy questions and hence generate different conclusions about the adequacy of $\mathrm{HRH}$ supply relative to requirements.

- Estimates of future HRH supply or requirements, however sophisticated, require numerous assumptions. As a number of the reviewed documents show, the estimated future HRH supplies and requirements are sensitive to differences in these assumptions. Even a few modest changes to these assumptions can mean the difference between a large estimated future shortage and a large 
estimated future surplus of HRH for a given jurisdiction and time period (e.g. [27, 105]).

To illustrate the implications of the above complexities, the substantial gaps in the available evidence, and the qualitatively and quantitatively different conclusions reached by different HRH analyses, Table 1 summarizes the results of the reviewed documents pertaining to Registered Nurses (RNs). Although several of these documents included multiple scenarios in their estimates, in the interests of a minimal level of comparability, the 'status quo' scenario is cited below unless otherwise stated.

As shown in Table 1, for the majority of included countries, no estimates of the size of any future RN shortage or surplus was found during this review. For the few jurisdictions for which such estimates were found, studies using different methods reached different conclusions. For example, Juraschek and colleagues [106] projected a shortage of over $900000 \mathrm{RNs}$ in the United States by 2030; meanwhile, the HRSA [41], using different methods and assumptions, estimated a surplus of over 300000 RNs for 2025.

A similar table for physicians would contain more information but no more clarity, since most analyses of physician supply and requirements focus on particular specialty areas such as emergency care [107] or obstetrics [108] as opposed to the physician supply as a whole. As Fig. 2 suggests, such a table for other professions would be even more sparsely populated. Virtually all documents reviewed seemed to suggest that HRH supply was expected to increase. Exceptions included, for example, nuclear medicine technologists in Australia [109], surgeons in Japan [66], obstetricians-gynaecologists in the United States [108], and RNs in Israel [110]. In contrast, as exemplified by the United States studies cited above, there are differing views about whether the requirements for different types of $\mathrm{HRH}$ are expected to increase or decrease in the future in different jurisdictions. Although most of the included analyses for nurses and physicians suggest that national-level shortages are likely in the next decade, several analyses for other nonphysician health professions such as pharmacists and dentists suggest substantial surpluses are likely over this time period (e.g. $[111,112])$. Should the scenarios depicted in these projections come to pass, the potential for substitution of different types of HRH would become an increasingly important policy issue; however, as noted above, most of the approaches identified through this review lack the capacity to account for such substitution.

\section{Knowledge gaps resulting from these limitations}

There has been a considerable amount of study devoted to the current and potential future states of the supply of and requirements for $\mathrm{HRH}$ in high-income OECD
Table 1 Projected national nurse shortages or surpluses by country for 2025

\begin{tabular}{|c|c|c|}
\hline Country & Document & $\begin{array}{l}\text { Projected } 2025 \text { shortage }(-) \\
\text { or surplus }(+)\end{array}$ \\
\hline \multirow[t]{3}{*}{$\overline{\text { Australia }}$} & $\begin{array}{l}\text { Health Workforce } \\
\text { Australia, 2012a }\end{array}$ & $\begin{array}{l}-109,400 \text { (includes RNs } \\
\text { and ENs) }\end{array}$ \\
\hline & $\begin{array}{l}\text { Health Workforce } \\
\text { Australia, 2012b }\end{array}$ & $-80,142$ \\
\hline & $\begin{array}{l}\text { Health Workforce } \\
\text { Australia, } 2014\end{array}$ & \multirow[t]{3}{*}{-85,000 (includes RNs and ENs) } \\
\hline Austria & No gap analyses found & \\
\hline Belgium & No gap analyses found & \\
\hline Canada & $\begin{array}{l}\text { Tomblin Murphy et al., } \\
2012\end{array}$ & \multirow[t]{7}{*}{$-60,000$} \\
\hline Chile & No gap analyses found & \\
\hline Czech Republic & No gap analyses found & \\
\hline Denmark & No gap analyses found & \\
\hline Estonia & No gap analyses found & \\
\hline Finland & No gap analyses found & \\
\hline France & No gap analyses found & \\
\hline Germany & $\begin{array}{l}\text { Maier and Afentakis, } \\
2013\end{array}$ & \multirow[t]{3}{*}{$\begin{array}{l}\text {-195,000 (includes RNs and ENs; } \\
\text { estimated from graph) }\end{array}$} \\
\hline Greece & No gap analyses found & \\
\hline Iceland & No gap analyses found & \\
\hline Ireland & $\begin{array}{l}\text { Training and } \\
\text { Employment } \\
\text { Authority, } 2009\end{array}$ & \multirow[t]{16}{*}{-836 (for year 2020) } \\
\hline Israel & No gap analyses found & \\
\hline Italy & No gap analyses found & \\
\hline Japan & No gap analyses found & \\
\hline Luxembourg & No gap analyses found & \\
\hline Netherlands & No gap analyses found & \\
\hline New Zealand & No gap analyses found & \\
\hline Norway & No gap analyses found & \\
\hline Poland & No gap analyses found & \\
\hline Portugal & No gap analyses found & \\
\hline Slovakia & No gap analyses found & \\
\hline Slovenia & No gap analyses found & \\
\hline Spain & No gap analyses found & \\
\hline South Korea & No gap analyses found & \\
\hline Sweden & No gap analyses found & \\
\hline Switzerland & No gap analyses found & \\
\hline United Kingdom & $\begin{array}{l}\text { Centre for Workforce } \\
\text { Intelligence, } 2013\end{array}$ & $\begin{array}{l}-50,000 \text { (for England only; } \\
\text { estimated from graph; } \\
\text { taken as midpoint of the } \\
\text { range of demand and supply } \\
\text { projection scenarios) }\end{array}$ \\
\hline \multirow[t]{3}{*}{ United States } & $\begin{array}{l}\text { Aiken and Cheung, } \\
2008\end{array}$ & $\begin{array}{l}-1,016,900 \text { (for year } 2020 ; \\
\text { analysis from another study) }\end{array}$ \\
\hline & Juraschek et al., 2012 & -918,232 (for year 2030) \\
\hline & HRSA, 2014 & $+340,000$ \\
\hline
\end{tabular}


countries. Despite these efforts, however, no clear or consistent picture of the status of these countries' HRH appears to exist. Some of the gaps in information are likely due to limitations in the various strategies used here to collect HRH analyses for this review. However, other recent studies, which did not have the same limitations (e.g. [6]), describe similar gaps in the information available. This is partly the result of significantly different approaches to HRH planning being used across and within these countries. The appropriateness of different $\mathrm{HRH}$ planning approaches for given jurisdictions depends on the objectives of the health care systems and the precise policy questions being asked for which they are planning as well as the context in which that planning takes place.

\section{A methodology for projecting $H R H$ requirements in high-income OECD countries}

The final objective of this review was to identify a methodology for projecting HRH requirements in highincome OECD countries. To guide the identification of such a methodology, a draft set of evaluation criteria was developed and circulated to the Advisory Group for their input, which was considered for the development of the list below:

1. The approach is consistent with the objectives of the health care system.

This means, for example, that a system whose objective involves addressing the health care needs of its population must use an HRH planning method that estimates $\mathrm{HRH}$ requirements as a function of population health measures so that resources can be planned in accordance with levels of -and potential changes in-the population's needs for health care. Resources are then allocated between populations based on differences in needs between those populations and increased or decreased over time in accordance with increases or decreases in those needs, while also allowing for changes in the way needs are to be met (e.g. using new technologies or different types of health care teams). Although meeting population health care needs is a goal shared by many health care systems, the findings of this review indicate that few countries appear to be using needs-based methods for HRH planning. Instead, those HRH analyses we found appear to be using utilization- or supply-based approaches. For countries such as the United States, where the ability to pay for health care services has traditionally been an important element of access to care, utilization-based approaches may be more relevant in addressing the policy questions facing decisionmakers. Although some of these approaches (e.g. [70]) incorporate consideration of how population health needs affect service use, this is done within the context of the prevailing organization of services in the United States and hence are unlikely to represent how needs for care would be served in the absence of payment at the point of delivery or via private insurance plans as a means of using care. For countries where meeting population health care needs is a primary objective, however, failure to plan for HRH according to those needs means planning not to meet that system objective. Moreover, the use of supply- or utilization-based approaches will perpetuate and exacerbate existing inefficiencies and inequalities in these systems $([2,113,114])$.

Needs-based approaches to $\mathrm{HRH}$ and health system planning are not new. Over 40 years ago, the WHO [115], for example, outlined a history of them dating back to at least the 1930s in the United States, also noting their widespread use in what are now former Soviet states. A more detailed description was later provided by Hall [116], and subsequently, such approaches were also described in the 1980s and 1990s in the United Kingdom [117, 118] and Canada [119-122]. Several more recent examples from Australia [53-56], Canada [2, 27, 57, 58, 104], and New Zealand [29, 59-61] were identified during this review.

2.

(a) $\mathrm{HRH}$ requirements are derived from service requirements, and

(b) Those service requirements are aligned with system objectives.

Requirements for $\mathrm{HRH}$ are a manifestation of requirements for the services they provide. Hence, estimates of $\mathrm{HRH}$ requirements must be derived from estimates of the requirements for those services. This makes it possible to consider and plan for potential future changes in the way services are delivered resulting from new technologies, changes in scopes of practice, and so on. The results of this review show, however, that $\mathrm{HRH}$ planning approaches that cannot account for such changes-such as the use of provider-population ratios-remain prevalent. This is unfortunate given that the major limitations of such approaches have been extensively documented for decades. For example, the WHO noted over 40 years ago that such approaches may be appropriate in,

...a country, whatever its stage of development, where professional judgement, backed by utilization studies, suggests that the current patterns of the organizations and delivery of health services [through] existing 
ratios do not present any anomalies or problems. Unfortunately, such situations are rare, and in any case this static view of society implies that the present situation cannot be significantly improved upon in the foreseeable future.

The exclusive use of health manpower: population ratios for the estimation of future health manpower requirements is increasingly difficult to justify in the developed countries where, depending on the specific characteristics of the health system, other and more refined criteria can be used to improve the country's capacity to provide health care. [115].

Other analyses found in this review use methods based on historical population-utilization ratios without any consideration of whether those ratios are consistent with the objectives of the health care system in question. The use of these methods for planning future services-and hence $\mathrm{HRH}$ - perpetuates any current patterns of inappropriate use such as over-reliance on emergency departments instead of primary health care or inadequate access to (and therefore use of) services by disadvantaged populations. The needs-based approaches from Australia, Canada, and New Zealand included in this review do not have this limitation. In addition, some 'needs-informed' but ultimately utilization-based approaches, such as those described by Dall and colleagues [70] and Gallagher and colleagues [86], are designed to explicitly account for potential changes to service provision as a consequence of, for example, changes in health care legislation or practice models.

3. The approach considers HRH requirements in the context of production functions for health services (i.e. dependent upon the availability or use of other inputs to service production).

Although the availability of $\mathrm{HRH}$ is important to the delivery of health care services, other types of human and non-human resources, such as facilities, equipment, and medications, are also necessary. Effective health systems planning approaches must recognize this dependency by considering how the availability (or lack thereof) of (a) other HRH and (b) non-human resources may affect their collective production of health care services, including the potential for substitution of one type of resource for another. For example, the availability of operating theatre nurses or operating theatres may impact on the volume of surgeries that surgeons can perform, even if the number of surgeons and the hours they work remain the same. The review found several examples of approaches that explicitly incorporated this potential for different types of $\mathrm{HRH}$ (e.g. $[29,53,123])$. However, although documents sometimes acknowledged the influence of the availability of non-human resources on $\mathrm{HRH}$ requirements, the review found no analyses that directly incorporated this relationship.

4. The approach explicitly considers the role and determinants of productivity (i.e. units of service per hour of work).

In order to translate health care service requirements into $\mathrm{HRH}$ requirements, $\mathrm{HRH}$ planners must consider the rate at which different types of $\mathrm{HRH}$ are able to provide those services per unit time-i.e. their productivity-under a given set of circumstances. In addition to the needs-based approaches referred to above, numerous other analyses found by the review (e.g. [25, 96, 124, 125]) explicitly included productivity as part of their calculations. Although the contexts in which productivity was considered varied widely across these documents, they generally showed that projections regarding the future $\mathrm{HRH}$ situation are highly sensitive to even small changes to $\mathrm{HRH}$ productivity.

5. HRH supply is measured in terms of time devoted to service delivery (i.e. flow generated by a stock of $\mathrm{HRH}$ ) as opposed to focusing only on the $\mathrm{HRH}$ stock (numbers of HRH).

The availability of health care services is determined by a number of factors in addition to the raw 'stock' or head count of different types of HRH available to provide them. Analyses in many countries have shown how changes or differences in these factors can have profound effects on the effective supply of HRH (e.g. [30, 66, 68]), and most of the HRH supply analyses found through the review considered at least one of these, most frequently hours worked. Several analyses (e.g. [96, 126, 127]), however, did not take any of these factors into account and instead estimated HRH supply based solely on head counts.

6. The approach considers the determinants of flow (e.g. hours worked) and stock (entries/exits) as policy variables.

The factors that determine the stock and flow of HRH supply, such as the amount of time spent providing patient care (activity levels), and the proportion of licensed HRH who are actively practising (participation levels), are sensitive-to varying degrees-to HRH policies such as education and payment models. Most of the analyses of $\mathrm{HRH}$ supply found through the review reflected this situation; in some cases, such factors were the primary focus of the analyses (e.g. [72, 78, 82]).

7. The approach considers the following: (a) The cost implications of $\mathrm{HRH}$ plans and 
(b) The extent to which HRH plans are aligned with health system financial planning

Essential to determining the relative appropriateness of any potential HRH policy is an understanding of its financial implications in the broader context of the jurisdictional fiscal situation. Although many of the documents included in the review acknowledged this point, comparatively few (e.g. [16, 76, 86, 128]) explicitly incorporated financial considerations into their analyses.

Figure 7 provides a summary illustration of how the various inputs into $\mathrm{HRH}$ planning that emerged across the reviewed documents would be addressed by an approach meeting these individual criteria.

Although over 200 documents discussing the HRH situations in the included countries were reviewed as part of this study, it appears that none of these approaches to HRH planning meets all seven of the above criteria. However, several of the approaches described above meet all but one. The needs-based approaches described in Australia [53-56], Canada [57, 58], and New Zealand [29, 59-61] each identify specific service requirements based on population health needs and translate these into $\mathrm{HRH}$ requirements based on information on scopes of practice and standards of care delivery. However, the Australian and New Zealand approaches do not appear to explicitly consider the role and determinants of productivity, while the Canadian approach does not include considerations of cost implications. Further, as noted above, none of the identified approaches appears to account for the impact of non-human resources on $\mathrm{HRH}$ requirements.

An example of a methodology for projecting $\mathrm{HRH}$ requirements in the included countries, then, could build on these needs-based approaches. This would require augmenting them in two ways. First, the Canadian approach would need additional features to include consideration of cost implications, and the Australian and New Zealand approaches would need to be further developed to include consideration of the role and determinants of productivity. Second, both approaches would need further enhancements to include consideration of the impacts of the availability of non-human resources.

The second of these two improvements is evidently the more difficult to achieve, as no HRH planning approaches found through this review included consideration of the impacts of non-human resources. The first of these improvements, however, has already begun. An analytical framework for such an approach has been developed by a team from Canada, Australia, and New Zealand [129] that extends existing needs-based methods to include consideration of the implications of $\mathrm{HRH}$ and other health system policies on the system's fiscal and socio-political sustainability. This framework has not yet been applied and was not included in the review because it was published after its specified time frame. Similar international collaborations may be one way of encouraging the continued advancement of $\mathrm{HRH}$ planning methods so that in the future they are also able to account for the impacts of non-human resources.

\section{Conclusions}

This review sought to synthesize the findings of the past decade of published research on HRH requirements and labour market dynamics in high-income OECD countries. Although over 200 documents pertaining to these topics were reviewed in detail, collectively, they do not include sufficient information to provide a clear picture of the expected future $\mathrm{HRH}$ situation in these countries. At best, it can be said that the HRH supply in these countries is generally expected to grow. Because different analyses reach different conclusions about future

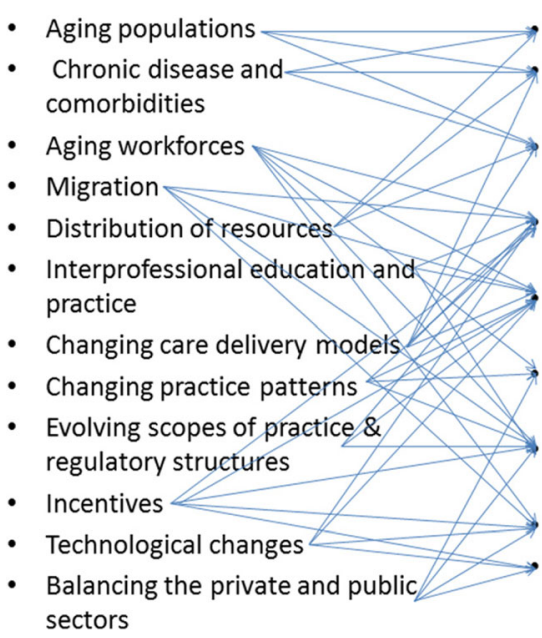

- Aging populations comorbidities

- Aging workforces

Migration

Distribution of resoun

Interprofessional education and practice

- Changing care delivery models

- Changing practice patterns

- Evolving scopes of practice \& regulatory structures

- Technological changes sectors
Consistent with system objectives

$\mathrm{HRH}$ requirements derived from service requirements

Service requirements derived from system objectives

Production functions for health services

Considers role \& determinants of productivity

Supply measured in terms of time devoted to service delivery

Considers determinants of stock and flow as policy variables

Considers costs

Considers alignment with financial planning

Fig. 7 Map of planning inputs to planning criteria 
$\mathrm{HRH}$ requirements in these countries, it is not clear whether that growth will be adequate to meet health care system objectives in the future. Although most analyses suggest that the numbers of physicians and nurses required in the included countries are likely to increase in the future, this view varies across analyses depending on the methods and assumptions used. Further, most analyses for professions other than nurses and physicians suggest that the numbers required are likely to decrease in the future. The implications of these projected respective surpluses and shortages in terms of meeting health care system objectives are not clear.

Several recurring themes regarding factors of importance in $\mathrm{HRH}$ planning were evident across the documents reviewed. These included the following: aging populations and health workforces; changes in disease patterns, models of care delivery, scopes of practice, regulatory structures, and technologies in health care; migration; incentives; data quality; the distribution of resources; interprofessional education and practice; stakeholder engagement; and balancing the public and private sectors. They also included important inconsistencies in the use of key HRH planning terms, which in turn affected the choice of methods in different documents for HRH planning.

Different approaches to HRH planning will be appropriate for different jurisdictions depending on their respective contexts and the objectives of their health care systems and hence the policy questions being faced. Based on these objectives, methods need to be adopted that produce relevant answers for the precise HRH questions facing policymakers in their particular contexts. The results of this review suggest, however, that rarely have explicit policy questions been identified to guide $\mathrm{HRH}$ research methods and analyses; instead, available methods have been adopted with policy 'interests' (as distinct from questions) made to fit these methods.

In an attempt to inform improvements to this situation, seven criteria for identifying an HRH planning approach appropriate to a given jurisdiction have been presented. Although none of the approaches found through the review met all of these criteria, several-from Australia, Canada, and New Zealand-met all but one. Examples of other approaches which met each individual criterion have also been identified so that planners can explore different options depending on the relative importance of these criteria given their respective circumstances and health system objectives.

Policymakers seeking to act on this evidence may consider multiple actions in the short term that may have short-, medium-, and long-term rewards. These include the following:

1. Identifying and engaging with the relevant $\mathrm{HRH}$ stakeholders in their respective jurisdictions to review and discuss the findings of this review and their implications for HRH planning in that jurisdiction. This may include consideration of the degrees to which

(a) The criteria for HRH planning approaches identified here are relevant for their jurisdiction,

(b) $\mathrm{HRH}$ planning approaches currently in use in their jurisdiction are consistent with those criteria, and

(c) HRH planning approaches currently in use in their jurisdiction could be improved by incorporating elements from others identified through this review.

2. Assessing the degree to which available data are adequate to inform $\mathrm{HRH}$ planning in that jurisdiction. Concerns about data are as long-standing as the study of HRH planning itself [115]. However, as has been noted elsewhere (e.g. [2]), problems with data are not avoided by relying on conceptually invalid models that ignore fundamental health care system objectives such as meeting population health needs by failing to incorporate measures of these or cannot account for potential future changes in factors such as productivity. It is better to base plans on appropriate concepts imperfectly measured than on inappropriate concepts that can be easily measured. If a particular jurisdiction's HRH stakeholders deemed the data available to them inadequate to fully inform planning, then investments should be made in improving the quality of the available data rather than in further entrenching the use of intrinsically flawed models. To that end, the identification and assessment of the data required to inform $\mathrm{HRH}$ planning should be based on the question of how many of what type of $\mathrm{HRH}$ are required to perform what services, for whom, and under what circumstances [2].

3. Items (1) and (2) above must be repeated on a regular basis as part of an iterative process for improving the alignment of $\mathrm{HRH}$ planning inputs and processes with the objectives of the jurisdiction's health care system.

\section{Additional files}

Additional file 1: List of websites searched. (DOC 52 kb)

Additional file 2: Data extraction tool for peer- and non-peer-reviewed literature. (DOC $32 \mathrm{~kb}$ )

Acknowledgements

The authors are grateful for the guidance provided by Jim Campbell and Giorgio Cometto of the World Health Organization's HRH unit, which also provided the funding for this work. The authors also acknowledge the contributions of the international Advisory Group whose members who were invited to provide supplementary documents to the review as well as input on the description of the methodology for estimating future $\mathrm{HRH}$ requirements. The members of the group were James Buchan, Jackie 
Cumming, Mario Dal Poz, Marjolein Dieleman, Gilles Dussault, Paulo Ferrinho, Maureen McCarty, Martine Morin, Guiliano Russo, Edward Salsberg, Tony Scott, and Kai Straehler-Poz.

\section{Authors' contributions}

GTM and SB led the conceptualization of the study; guided the development of the review parameters, the collection and analyses of the documents, and the reporting of the findings; led the development of the $\mathrm{HRH}$ planning criteria; and contributed to the writing of the manuscript. AM contributed to the conceptualization of the study as well as the development of the review criteria and the $\mathrm{HRH}$ planning criteria and led the collection and analyses of the documents, reporting of the findings, and writing of the manuscript. SB contributed to the development of the review parameters, the collection and analyses of the documents, and the writing of the manuscript. AER contributed to the collection and analyses of the documents and the writing of the manuscript. All authors read and approved the final manuscript.

\section{Competing interests}

The authors declare that they have no competing interests. Although several papers by some of the authors were among those included in the review, we have endeavoured to discuss all the included papers with equal impartiality.

\section{Author details}

'Dalhousie University, 5869 University Avenue, Halifax, NS B3H 4R2, Canada. ${ }^{2}$ McMaster University, 1280 Main Street West, Hamilton, ON L8S 4K1, Canada.

\section{Received: 23 December 2015 Accepted: 13 September 2016} Published online: 29 September 2016

\section{References}

1. Mejía A, Fülöp T. Health manpower planning: an overview. In: Hall T, Mejía A, editors. Health manpower planning: principles, methods, issues. Geneva: WHO; 1978. p. 9-30.

2. Birch S, Kephart G, Tomblin Murphy G, O'Brien-Pallas L, Alder R, MacKenzie A. Human resources planning and the production of health: a needs-based analytical framework. Canadian Public Policy. 2007;33(Supplement):S1-S16.

3. Campbell J, Dussault G, Buchan J, Pozo-Martin G, Martin F, Guerra Arrias M, et al. A universal truth: no health without a workforce. Recife, Brazil. Geneva: Report on the Third Global Forum on Human Resources for Health; 2014.

4. Matrix Insight. EU level Collaboration on Forecasting Health Workforce Needs, Workforce Planning and Health Workforce Trends - A Feasibility Study. Brussels: European Commission; 2007

5. Dussault G, Buchan J, Sermeus W, Padaiga Z. Assessing future health workforce needs. Copenhagen: WHO; 2010.

6. Ono $T$, Lafortune $G$, Schoenstein $M$. Health workforce planning in OECD Countries: a review of 26 projection models from 18 countries. OECD Health Working Papers. 2013; 62: 1-130.

7. World Health Organization Secretariat. Draft global strategy on human resources for health: Workforce 2030. Geneva: WHO; 2016.

8. Dal Poz M, Dreesch N, Fletcher S, Gedik G, Gupta N, Hornby PSD. Models and tools for health workforce planning and projections. Human Resources for Health Observer. 2010; 3:1-17.

9. Anand S. Measuring health workforce inequalities: methods and application to China and India. Human Resources for Health Observer. 2010; 5:1-42.

10. Scheffler RM, Bruckner T, Spetz J. The labour market for human resources for health in low and middle-income countries. Human Resources for Health Observer. 2012;10: 1-20.

11. Scottish Executive. Health workforce plan 2006. Edinburgh: Scottish Executive; 2006

12. McPake B, Maeda A, Araujo EC, Lemeire C, El Maghraby A, Cometto G. Why do health labour market forces matter? Bull World Health Organ. 2013;91: 841-6.

13. Masnick K, McDonnell G, Masnick K, McDonnell G. A model linking clinical workforce skill mix planning to health and health care dynamics. Hum Resour Health. 2010:8:11.

14. United States Health Resources and Services Administration (HRSA). Health workforce projections: Respiratory Therapists. Washington: US Department of Health and Human Services; 2015.
15. Centre for Workforce Intelligence. A strategic review of the future healthcare workforce: Informing medical and dental student intakes. London: CfWl; 2012.

16. Tennant M, Kruger E. Turning Australia into a 'flat-land': what are the implications for workforce supply of addressing the disparity in rural-city dentist distribution. Int Dent J. 2014;64:29-33.

17. Kuhlmann E, Batenburg R, Groenewegen PP, Larsen C. Bringing a European perspective to the health human resources debate: a scoping study. Health Policy. 2013;110:6-13.

18. Fellows J, Lyscom T, Burge C, Edwards M. Horizon 2035: International responses to big picture challenges. London: Centre for Workforce Intelligence; 2014

19. OECD. Population age structure. Paris:OECD; 2015

20. Keefe J, Légaré J, Carrière Y. Developing new strategies to support future caregivers of older Canadians with disabilities: projections of need and their policy implications. Canadian Public Policy. 2007; 33(Supplement):S66-80.

21. Salsberg E, Erickson C. The changing physician workforce landscape-implications for physical medicine and rehabilitation. American Journal of Physical Medical Rehabilitation. 2007;86(10):838-44.

22. Tomblin Murphy G, Kephart G, Lethbridge L, O'Brien-Pallas L, Birch S. Planning for what? Challenging the assumptions of health human resources planning. Health Policy. 2009;92(2-3):225-33.

23. Birch S, Mason T, Sutton M, Whittaker W. Not enough doctors or not enough needs? Refocusing health workforce planning from providers and services to populations and needs. Journal of Health Services Research and Policy. 2013;18(2):107-13.

24. Mason T, Sutton M, Whittaker W, Birch S. Exploring the limitations of agebased models for health care planning. Soc Sci Med. 2015;132:11-9.

25. Buske L. Projections of physician supply in Canada. Ottawa: Canadian Medical Association; 2007.

26. Helsedirektoratet, World Health Organization, Global Health Workforce Alliance. Consultation on human resources for health for high income countries. Preparatory Meeting for the Third Global Forum on HRH: Meeting Report. Geneva: WHO; 2013

27. Tomblin Murphy G, Birch S, MacKenzie A, Alder R, Lethbridge L, Little L. Eliminating the shortage of registered nurses in Canada: an exercise in applied needs-based planning. Health Policy. 2012;105:192-202.

28. Organisation for Economic Co-operation and Development. The looming crisis in the health workforce: How Can OECD Countries Respond? Paris: OECD; 2008

29. New Zealand Ministry of Health. Aged care workforce service review. Wellington: New Zealand Ministry of Health; 2011.

30. Johannessen KA, Hagen TP. Variations in labor supply between female and male hospital physicians: results from a modern welfare state. Health Policy. 2012:107:74-82

31. Buchan J. Achieving workforce growth in UK nursing: policy options and implications. Collegian. 2009:16:3-9.

32. Ognyanova D, Busse R. A destination and a source: Germany manages regional health workforce disparities with foreign medical doctors. In: Wismar M, Maier CB, Glinos IA, Dussault G, Figueras J, editors. Health professional mobility and health systems: evidence from 17 European countries. Copenhagen: World Health Organization; 2011. p. 211-42.

33. Longley M, Riley N, Davies P, Hernández-Quezvedo C. United Kingdom (Wales) health system review. Health Systems in Transition. 2012;14:11.

34. Pantelli D, Sagan A, Busse R. Poland health system review. Health Systems in Transition. 2011:13:8

35. Sigurgeirsdóttir S, Waagfjörð J, Maresso A. Iceland health system review. Health Systems in Transition. 2014;16:6.

36. Gaál P, Szigeti S, Csere M, Gaskins M, Panteli D. Hungary health system review. Health Systems in Transition. 2011;13:5.

37. Health Workforce Australia. Australia's future health workforce-doctors. Canberra:; 2014.

38. Cooper RA. The US physician workforce, where do we stand? Paris: OECD 2008

39. Royal Australasian College of Surgeons. Surgical workforce projections to 2025 (for Australia). Melbourne: RACS; 2011.

40. Aiken LH. U.S. nurse labor market dynamics are key to global nurse sufficiency. Health Serv Res, 2007:42(3):1299-320.

41. United States Health Resources and Services Administration (HRSA). The future of the nursing workforce. Washington: National- and State-Level Projections, 2012-2025; 2014. 
42. Toyokawa S, Kobayashi Y. Increasing supply of dentists induces their geographic diffusion in contrast with physicians in Japan. Soc Sci Med. 2010;71:2014-9.

43. Chevreul K, Durand-Zaleski I, Bahrami S, Hernández-Quevedo C, Mladovsky P. France health system review. Health Systems in Transition. 2010;12:6.

44. Hawthorne N, Anderson C. The global pharmacy workforce: a systematic review of the literature. Hum Resour Health. 2009;7:48.

45. Ide H, Yasunaga HKT, Koike S, Taketani Y, Imamura T. The dynamics of obstetricians and gynecologists in Japan: a retrospective cohort mode using the nationwide survey of physicians data. Journal of Obstetrics \& Gynaecology Research. 2009;35(4):761-6.

46. Olejaz M, Nielsen AJ, Rudkjøbing A, Okkels Birk H, Krasnik A, Hernández-Quevedo C. Denmark health system review. Health Systems in Transition. 2012;14:2.

47. Anell A, Glenngård AHMS. Sweden health system review. Health Systems in Transition. 2012;14:5

48. Ishikawa T, Ohba H, Yokooka Y, Nakamura K, Ogasawara K. Forecasting the absolute and relative shortage of physicians in Japan using a system dynamics model approach. Hum Resour Health. 2013;11:14.

49. Dubois CA, Singh D. From staff-mix to skill-mix and beyond: towards a systemic approach to health workforce management. Hum Resour Health. 2009;7(87):1-19.

50. Scott A, Sivey P, Joyce C, Schofield D, Davies P. Alternative approaches to health workforce planning. Adelaide: National Health Workforce Planning and Research Collaboration; 2011.

51. European Commission. Commission staff working document on an action plan for the EU health workforce. Strasbourg: European Commission; 2012.

52. New York Health Workforce Data System, Center for Health Care Workforce Studies. The health care workforce in New York: Trend s in the supply and demand for health workers. Resselaer: Center for Health Care Workforce Studies; 2014.

53. Andrews $G$, Titov $N$. Changing the face of mental health care through needs-based planning. Aust Health Rev. 2007;31(Supplement):S122-8.

54. Segal L, Dalziel K, Bolton T. A work force model to support the adoption of best practice care in chronic diseases - a missing piece in clinical guideline implementation. Implement Sci. 2008;3:35.

55. Segal L, Leach MJ. An evidence-based health workforce model for primary and community care. Implement Sci. 2011;6:93.

56. Segal $L$, Leach MJ, May E, Turnbull C. Regional primary care team to deliver best-practice diabetes care: a needs-driven health workforce model reflecting a biopsychosocial construct of health. Diabetes Care. 2013;36(7):1898-907.

57. Tomblin Murphy G, MacKenzie A, Alder R, Langley J, Hickey M, Cook A. Pilot-testing an applied competency-based approach to health human resources planning. Health Policy and Planning. 2013;28(7):739-49.

58. Tomblin Murphy G, MacKenzie A, Rigby J, Rockwood K, Gough AGA, Montpetit $F$, et al. Service-based health human resources planning for older adults. J Am Med Dir Assoc. 2013;14(8):611-5.

59. New Zealand Ministry of Health. Diabetes workforce service review. Wellington: New Zealand Ministry of Health; 2011.

60. New Zealand Ministry of Health. Mental health and addictions workforce service forecast. Wellington: New Zealand Ministry of Health; 2011.

61. New Zealand Ministry of Health. Dermatology workforce service forecast Wellington:New Zealand Ministry of Health; 2014.

62. Ireland Health Service Executive. Medical workforce planning: Second interim project report. Dublin: Ireland Health Service Executive; 2014.

63. New Zealand Ministry of Health. Health workforce development: An overview. Wellington: New Zealand Ministry of Health; 2006.

64. Health Education England. Workforce plan for England: Proposed Education and Training Commissions for 2014/15. London: Health Education England; 2013.

65. Health Workforce Australia. Health workforce 2025, vol. 1: Doctors, Nurses and Midwives. Adelaide: Health Workforce Australia; 2012

66. Koike S, Matusmoto S, Kodama T, Ide H, Yasunaga HIT. Estimation of physician supply by specialty and the distribution impact of increasing female physicians in Japan. BMC Health Serv Res. 2009:9:180.

67. Goodyear-Smith F, Janes R. New Zealand rural primary health care workforce in 2005: more than just a doctor shortage. Aust J Rural Health. 2008;16:40-6.

68. Crossley TF, Hurley J, Jeon SH. Physician labour supply in Canada: a cohort analysis. Health Econ. 2009;18:437-56.

69. Stordeur S, Leonard C. Challenges in physician supply planning: the case of Belgium. Hum Resour Health. 2010;8:28.
70. Dall T, West T, Chakrabati R, lobucci W. The complexities of physician supply and demand: projections from 2013 to 2035. Washington: Association of American Medical Colleges; 2015.

71. Spetz J. How will health reform affect demand for RNs? Nurs Econ. 2014;32(1):42-4.

72. McPake B, Scott A, Edoka I. Analyzing markets for health workers. Washington: World Bank; 2014.

73. Campbell J, Buchan J, Cometto G, David B, Dussault G, Fogstad H, et al. Human resources for health and universal health coverage: fostering equity and effective coverage. Bull World Health Organ. 2013;91:853-63.

74. Stange K. How does provider supply and regulation influence health care markets? Evidence from nurse practitioners and physician assistants. J Health Econ. 2014;33:1-27.

75. Dubois CA, McKee M, Nolte E. Human resources for health in Europe. Berkshire: Open University Press; 2006.

76. Baltagi $\mathrm{BH}$, Bratberg $\mathrm{E}$, Helge HT. A panel data study of physicians' labor supply: the case of Norway. Health Econ. 2005;14:1035-45.

77. Cox A, Grimshaw D, Carroll M, McBride A. Reshaping internal labour markets in the National Health Service: new prospects for pay and training for lower skilled service workers. Hum Resour Manag J. 2008;18(4):347-65.

78. McRae I, Butler JRG. Supply and demand in physician markets: a panel data analysis of GP services in Australia. Int J Health Care Finance Econ. 2014;14:269-87.

79. Buchan J, Aiken LH. Solving nursing shortages: a common priority. J Clin Nurs. 2008;17(24):3262-8

80. Buchan J, O'May F, Dussault G. Nursing workforce policy and the economic crisis: a global overview. J Nurs Scholarsh. 2013;46(3):298-307.

81. Marvasti A. An estimation of the demand and supply for physicians services using a panel data. Econ Model. 2014;43:279-85.

82. Jeon SH, Hurley J. Physician resource planning in Canada: the need for a stronger behavioural foundation. Canadian Public Policy. 2010;36(3):359-75.

83. Buchan J, Black S. The impact of pay increases on nurses' labour market: a review of evidence from four OECD countries. Paris: OECD; 2011.

84. World Health Organization Western Pacific Office. WPRO workforce projection tool version 1.0 user's manual. Manila: WHO Western Pacific Office; 2008

85. World Health Organization. Workload indicators of staffing need (WISN) software manual. Geneva: WHO; 2010.

86. Gallagher JE, Kleinman ER, Harper PR. Modelling workforce skill-mix: how can dental professionals meet the needs and demands of older people in England? Br Dent J. 2010;208:E6.

87. Spetz J, Dyer WT, Chapman S, Seago JA. Hospital demand for licensed practical nurses. West J Nurs Res. 2006;28(6):726-39.

88. Centre for Workforce Intelligence. A strategic review of the future pharmacist workforce: informing pharmacist student intakes. London: CfWl; 2013.

89. Arnhold M, Quade MKW. Mobile applications for diabetics: a systematic review and expert-based usability evaluation considering the special requirements of diabetes patients age 50 years or older. Journal of Medical Internet Research. 2014;16(4):e104.

90. Baumann A, Kolotylo C. Forecasting future workforce demand: a process evaluation. Hamilton: Nursing Health Services Research Unit: 2010.

91. Oakley P, Ngo S, Vinten M, Budjanovcanin A. NHS Wales: midwifery workforce planning project. Cardiff: Developing a Workforce Planning Model; 2008.

92. Centre for Workforce Intelligence. Horizon scanning-a strategic review of the future healthcare workforce. London: CfWl; 2013.

93. Crettenden IF, McCarty M, Fenech BJ, Heywood T, Taitz MC, Tudman S. How evidence-based workforce planning in Australia is informing policy development in the retention and distribution of the health workforce. Hum Resour Health. 2014;12:7.

94. Holmes M. Developing an open source simulation model of physician supply and healthcare utilization. Washington: Association of American Medical Colleges Workforce Conference; 2012

95. Crescent Management Consulting. New Brunswick health human resources supply and demand update 2008-2015. Fredericton: New Brunswick Department of Health; 2009.

96. Barber P, González López-Valcárcel B. Forecasting the need for medical specialists in Spain: application of a system dynamics model. Hum Resour Health. 2010;8:24.

97. Chaloff J. Immigration of Health Professionals to Italy: Mismatches in the formal sector, expansion of the informal sector. Paris: OECD; 2008. 
98. Takata $H$, Nagata $H$, Nogawa $H$, Tanaka $H$. The current shortage and future surplus of doctors: a projection of the future growth of the Japanese medical workforce. Hum Resour Health. 2011;9:14.

99. United States Health Resources and Services Administration (HRSA). Technical documentation for HRSA's health workforce simulation model. Rockville: US Department of Health and Human Services; 2014.

100. Van Greuningen M, Batenburg RS, Van der Velden LFJ. Ten years of health workforce planning in the Netherlands: a tentative evaluation of GP planning as an example. Hum Resour Health. 2012;10:21.

101. Van Greuningen M, Batenburg RS, Van der Velden LFJ. The accuracy of general practitioner workforce projections. Hum Resour Health. 2013;11:31.

102. Centre for Workforce Intelligence. Horizon scanning: Analysis of fac tors and forces. London: CfWl; 2014

103. Centre for Workforce Intelligence. Elicitation methods: Applying elicitation methods to robust workforce planni ng. London: CfWl; 2015.

104. Singh D, Lalani H, Kralj B, Newman E, Goodyear J, Hellyer D, et al. Ontario population needs-based physician simulation model. Toronto: Ontario Ministry of Health and Long-Term Care; 2010.

105. Centre for Health Workforce Intelligence. Future midwifery workforce projections: Starting the discussion. London: CfWl; 2013.

106. Juraschek SP, Zhang X, Ranganathan VK, Lin VW. United States registered nurse workforce report card and shortage forecast. Am J Med Qual. 2012;27(3):241-9.

107. Sullivan AF, Ginde AA, Espinola JA, Camargo CA. Supply and demand of board-certified emergency physicians by U.S. state, 2005. Acad Emerg Med. 2009;16(10):1014-8.

108. Anderson BL, Hale RW, Salsberg E, Sculkin J. Outlook for the future of the obstetrician-gynecologist workforce. American Journal of Obstetrics \& Gynecology. 2008;199(1):e1-8.

109. Adams E, Schofield D, Cox J, Adamson B. Will the Australian nuclear medicine technologist workforce meet anticipated health care demands? Aust Health Rev. 2008;32(2):282-91.

110. Nirel N, Riba S, Reicher S, Toren O. Registered nurses in Israel—workforce employment characteristics and projected supply. Israel Journal of Health Policy Research. 2012;1(1):11.

111. United States Health Resources and Services Administration (HRSA). Health workforce projections: Pharmacists. Washington: US Departent of Health and Human Services; 2015.

112. United States Health Resources and Services Administration (HRSA). National and state-level projections of dentists and dental hygienists in the U.S., 2012-2025. Washington:US Department of Health and Human Services; 2015.

113. Evans RG. There's no reason for it, it's just our policy. Healthcare Policy. 2009; 5(2):14-24.

114. Brocklehurst $P$, Tickle M. Planning a dental workforce for the future for the National Health Service in the United Kingdom: what factors should be accounted for? Health Educ J. 2011;71(3):340-9.

115. World Health Organization. The development of studies in health manpower: Report of a WHO scientific working group. Geneva: WHO; 1971.

116. Hall T. Demand. In: Hall T, Mejía A, editors. Health manpower planning: principles, issues, methods. Geneva: WHO; 1978. p. 57-90.

117. Birch S. Equity and efficiency in medical manpower planning: defining objectives and looking towards the future. Health Policy. 1985;4:341-6.

118. Birch S, Maynard A. Dental manpower. Social Policy \& Administration. 1985; 19(3):199-217

119. Eyles J, Birch S, Chambers S, Hurley J, Hutchinson B. A needs-based methodology for allocating health care resources in Ontario. Canada: development and an application. Social Science \& Medicine. 1991;33:4.

120. Birch S, Chambers S. To each according to need: a community-based approach to allocating health care resources. Can Med Assoc J. 1993;149(5):607-12.

121. Newbold KB, Eyles J, Birch S, Spencer A. Allocating resources in health care: alternative approaches to measuring needs in resource allocation formula in Ontario. Health and Place. 1998;4(1):79-89.

122. Birch S, Eyles J, Hurley J, Hutchinson B, Chambers S. A needs-based approach to resource allocation in health care. Canadian Public Policy. 1993;19(1):68-85.

123. Scottish Government. An analysis of the dental workforce in Scotland. Edinburgh: NHS Education for Scotland; 2010.

124. Artoisenet $C$, Deliège D. Medical workforce in Belgium: assessment of future supply and requirements. Louvain Médical. 2006;125(1):4-21.

125. Dill MJ, Salsberg E. The complexities of physician supply and demand: projections through 2025. Washington: Association of American Medical Colleges; 2008.
126. Capaciteits Orgaan. The 2013 Recommendations for medical specialist training. Utrecht: Capaciteits Orgaan; 2013.

127. Landry MD, Ricketts TC, Verrier MC. The precarious supply of physical therapists across Canada: exploring national trends in health human resources (1991 to 2005). Hum Resour Health. 2007:5:23.

128. Polgreen LA, Mott DA, Doucette WR. An examination of pharmacists' labor supply and wages. Research in Social \& Administrative Pharmacy. 2011;7:406-14.

129. Birch S, Tomblin Murphy G, MacKenzie A, Cumming J. In place of fear: aligning health care planning with system objectives to achieve financial sustainability. Journal of Health Services Research \& Policy. 2015;20(2):109-14.

\section{Submit your next manuscript to BioMed Central and we will help you at every step:}

- We accept pre-submission inquiries

- Our selector tool helps you to find the most relevant journal

- We provide round the clock customer support

- Convenient online submission

- Thorough peer review

- Inclusion in PubMed and all major indexing services

- Maximum visibility for your research

Submit your manuscript at www.biomedcentral.com/submit
) Biomed Central 